\title{
Evaluation of Dry Eye Syndrome in Patients With Familial Mediterranean Fever: A Cross-sectional Study
}

\author{
Senem Şaş, M.D. ${ }^{1}$, Ali Kurt, M.D. ${ }^{2}$, Emine Eda Kurt, M.D. ${ }^{3}$, Fatmanur Aybala Koçak, M.D. ${ }^{3}$, \\ Hatice Rana Erdem, M.D. ${ }^{3}$ \\ ${ }^{1}$ Division of Rheumatology, Department of Physical Medicine and Rehabilitation, Erciyes University Faculty of Medicine, Kayseri, ${ }^{2}$ Department of \\ Ophtalmology, ${ }^{3}$ Department of Physical Medicine and Rehabilitation, Kırşehir Ahi Evran University Faculty of Medicine, Kırşehir, Turkey
}

\begin{abstract}
Objective. It has been reported that some autoimmune diseases are associated with dry eye syndrome. There are limited studies that relates dry eye syndrome in patients with Familial Mediterranean fever (FMF). The aim of this study is to evaluate the relationship between dry eye syndrome in patients with FMF comparing with healthy controls. Methods. Prospective cross-sectional study was carried out in departments of physical medicine and rehabilitation and ophthalmology clinics in a tertiary public institution with a sample of 46 participants including 23 FMF patients and 23 healthy controls. Detailed eye examination was performed in all cases and indicators of xerophthalmia were assessed by Schirmer-I test, tear break-up time (TBUT) and ocular surface disease index (OSDI). In patient group; results of FMF gene analysis, acute phase reactants, concomitant diseases and number of FMF attacks were noted. Results. The average Schirmer-I test results for right-eye were recorded as $14.74 \pm 8.38$ for the FMF group and $13.09 \pm 10.54$ for the healthy group; the TBUT scores were $14.69 \pm 5.32$ in FMF group and $15 \pm 6.45$ in the healthy group; and OSDI scores were $9.04 \pm 2.75$ and $10.86 \pm 1.77$ in the FMF group and the healthy control, respectively. There were no statistically significant differences between FMF and control groups in terms of Schirmer-I, TBUT, and OSDI scores (all $\mathrm{p}>0.05$ ). No correlation was identified between acute phase reactants and the OSDI, TBUT and Schirmer-I tests for right-eye (all $p>0.05$ ). Conclusion. This study identified no correlation between dry eye and FMF. Ocular pathology is rare and infrequently reported in FMF. (J Rheum Dis 2020;27:270-276)
\end{abstract}

Key Words. Familial mediterranean fever, Dry eye syndrome, Inflammation, Xerophthalmia

\section{INTRODUCTION}

Familial Mediterranean fever (FMF) is the most frequent autoinflammatory disease and characterized by recurrent polyserositis and fever that is lasting one to four days. It is inherited by autosomal recessively [1] and its prevalence is $1 / 500$ in the Jewish and Armenian ethnicities, and 1/1,000 in Turkish and 1/25 in Arabic ethnicities $[2,3]$. FMF attacks usually involve joint, pleura, peritoneum or the skin. In most cases, FMF attacks lasts for one to three days. Inflammation episodes related to FMF are mediated by a massive penetration of neutrophils into serous cavities and result in elevated acute-phase reactants and cytokines. FMF patients remain symptom-free between the attacks. In the pathophysiology of FMF, mutation in the Mediterranean fever (MEFV) gene located in 16th chromosome has been found to be responsible [1-3].

Many systems can be involved in FMF. In most cases, oral colchicine treatments are enough to ensure remission [1-3]. Ocular involvement is rare but has been referenced in case reports related to episcleritis, keratitis and uveitis (anterior, intermediate, posterior and panuveitis) in FMF [4-7].

Dry eye syndrome (DES) is a multifactorial ocular surface disease of the tear ducts that leads to discomfort and

Received : May 18, 2020, Revised : July 1, 2020, Accepted : July 20, 2020

Corresponding to : Senem Şaş (iD http://orcid.org/0000-0002-5616-5723

Division of Rheumatology, Department of Physical Medicine and Rehabilitation, Erciyes University Faculty of Medicine, Köşk Mah. Talas Bulvarı. No:1, Kayseri 38030,Turkey. E-mail : senemsas@gmail.com

Copyright (c) 2020 by The Korean College of Rheumatology.

This is an Open Access article, which permits unrestricted non-commerical use, distribution, and reproduction in any medium, provided the original work is properly cited. 
results in visual disorders. Keratoconjunctivitis sicca, as a synonym of DES, is characterized by potential damage to the ocular surface. Since the eye is a target of inflammation, many inflammatory diseases are associated with ocular involvement. In autoimmune diseases, the involvement of the lachrymal gland leads to lymphocyte infiltration. Inflammatory cytokines impair the functions of the lachrymal gland and cause low tear production [8]. $\mathrm{T}$ helper cell 17 (Th-17) increases cytokines and inflammation in autoimmune diseases and it has been demonstrated that serum levels of tumor necrosis factor- $\alpha$ (TNF- $\alpha$ ), interleukin-6 (IL-6), IL-8, soluble IL-2 receptors and soluble receptors of TNF- $\alpha$ (p55 and p75 receptors) increase during FMF attacks, although increased serum TNF- $\alpha$ levels are also noted in symptom-free periods $[9,10]$. As inflammatory cytokines, IL-6 and IL-8 have also been determined in DES status and IL-1 receptor antagonists have been shown to be elevated in DES-related disease. In addition, while DES upregulates lymphocytes, in FMF elevated levels of neutrophils are measured in attack [11,12]. Moreover, IL-1 receptor blockade have been shown treatment of DES [13]. Also IL-1 receptor blockage is the leading treatment for colchicine resistance [14].

DES usually accompanies such systemic inflammatory diseases as Rheumatoid Arthritis (RA) and Sjögren's syndrome $[8,15]$. In the literature, there are only limited studies that investigated DES in patients with FMF. In a study by Karalezli et al. [16] tear-film functions using were evaluated by Schirmer-I, break-up time (BUT), corneal fluorescein and Rose Bengal tests. They also had evaluated ocular surface changes by determining cell content of surface conjunctival epithelium using conjunctival impression cytology. According to the results of that study, it was reported that despite normal tear production, the ocular surface and tear-film functions of FMF patients differ from those of healthy individuals.

Since there are few data in the literature, the present study aims to evaluate the presence of dry eye in patients with FMF who were followed with musculoskeletal complaints by Physical Medicine and Rehabilitation (PMR) outpatient clinic.

\section{MATERIALS AND METHODS}

Twenty-three patients with FMF who presented to the PMR outpatient clinics of Ahi Evran University Training and Research Hospital and 23 healthy individuals were included in the study. The present study compared the dry eye profile between patients with FMF and healthy controls. Before the study, permission was obtained from the local ethics committee (approval number: 2017-09/73, Date: 23.05.2017). The participants were informed about the aim and method of the study and their written and oral consent was obtained. This study was conducted in accordance with the Helsinki Declaration.

\section{Participant selection}

FMF patients who were diagnosed according to the Tel-Hashomer criteria was the study group [17]. All of the FMF patients were referred from the PMR outpatient clinics. The control group was consisted of healthy people from hospital staff who were age and sex matched with the FMF patient group. Ocular (i.e., blepharitis) or systemic infections, contact lens use, prevalent intraocular surgery, cigarette and alcohol consumption, tumors, hypertension, thyroid function disorders, diabetes mellitus, hepatic and renal failure, hemorrhagic diseases, serious anemia, pregnancy, psychiatric disorders and the use of systemic or topical medication that could affect the tear-film or meibomian gland functions ( $\beta$-Blocker, antihistaminic, corticosteroids, antihypertensive etc.) causing DES symptoms were identified as exclusion criteria for both groups. The patients and controls who had communication problems (dementia or hearing loss); a history of ocular surface disorder (Pemphigoid, Sjögren's syndrome or any other type of conjunctivitis or keratitis), previous ocular surgery or ocular injury and topical eye medication use within the previous 3 months; were also excluded from the study.

\section{Evaluation parameters}

1) Demographic and disease specific clinical features All of the FMF patients were examined by the same physician in the outpatient clinic of PMR department. All of the FMF patients were questioned regarding some demographic and disease spesific clinical data, including age, gender, MEFV gene mutation, disease duration, time of the diagnosis, number of recurrences of attacks, the presence of systemic disease, previous therapies and eye complaints. The assessment of the clinical condition of the patients was made via routine blood sample tests, including a complete blood count, erythrocyte sedimentation rate (ESR), C-reactive protein level (CRP), fibrinogen level, fasting blood glucose level, hepatic and renal func- 
tion tests and urine analysis.

\section{2) Ophtalmologic examination}

All of the patients and healthy volunteers underwent an ophthalmological examination by the same ophthalmologist. The right eye of each participant was studied, with a detailed ocular examination that included intraocular pressure, slit-lamp bio microscopy, dilatation of fundus and xerophthalmia tests, which are TBUT (without anesthesia) and Schirmer-I test. Also OSDI questionnaire was applied to participants.

The TBUT test was performed with a strip of fluorescein moistened with distilled water, carried out on the inferior conjunctiva sachet. The precordial tear film was observed under the cobalt blue filter of the slit-lamp bio microscope and the time between the last blink and the appearance of the first dry spot on the cornea was recorded. This process was repeated three times and the average time values were noted in seconds. DES is diagnosed in cases where TBUT is less than 10 seconds [18].

The Schirmer-I test was carried out using standard filter-paper strips (Whatmann, Maidstone, UK). The strips were placed on the lateral fornix of the cases and the average measurement of the wet strips after 5 minutes was noted as the Schirmer-I value. The tests lasted for at least 15 minutes, although no examinations were carried out that could lead to reflex eye [18].

OSDI, which is a specific questionnaire that examines the quality of life, contains 12 questions that assess ocu- lar discomfort, environmental triggers and functioning [19]. The time interval of the questionnaire covers the previous week and the participants are asked to reply to each question with never, occasionally, half of the time, most of the time or all the time. The range of possible OSDI scores is $0 \sim 100$ intervals, with higher scores indicating greater disability. DES is diagnosed when OSDI scores are $>15$. The Turkish reliability and validity study of the OSDI was done by Irkec et al. [20].

\section{Statistical analyses}

The statistical analyses were made using IBM Statistical Package for the Social Sciences (SPSS) version 20.0 software (IBM Corporation, Armonk, NY, USA) and data was presented as the arithmetic mean and standard deviation, while categorical measurements were presented as percentages (\%). A Shapiro-Wilks test was used to evaluate normal distribution. When the data was normally distributed in the comparison of the two groups, a Student's t-test was carried out; and when the data was not normally distributed, a Mann-Whitney U-test was applied. A chi-square test was used to compare qualitative data and Spearman correlation tests were used for the analysis of the data. The statistical level of significance was accepted as $\mathrm{p}<0.05$.

The $G^{*}$ Power package software program ( $G^{*}$ Power, version 3.1.9.2; Franz Faul, University of Kiel, Kiel, Germany) and a two-sample test was used to determine the sample size and it was calculated that a sample of at

Table 1. The demographic and clinical features of the patients and healthy controls

\begin{tabular}{|c|c|c|c|}
\hline Variable & $\begin{array}{l}\text { FMF patients } \\
\qquad(\mathrm{n}=23)\end{array}$ & $\begin{array}{l}\text { Healthy controls } \\
\qquad(\mathrm{n}=23)\end{array}$ & p-value \\
\hline Age $(y r)$ & $30.96 \pm 10.31$ & $30.96 \pm 9.51$ & 0.848 \\
\hline Sex (female/male) & $12 / 11(52.2 / 47.8)$ & $15 / 8(65.2 / 34.8)$ & 0.235 \\
\hline Disease duration (yr) & $19.08 \pm 12.40$ & & \\
\hline Time of the diagnosis $(y r)$ & $8.19 \pm 9.34$ & & \\
\hline Attack frequency (per yr) & $4.08 \pm 4.01$ & & \\
\hline \multicolumn{4}{|l|}{ MEFV gene mutation } \\
\hline M694V homozygote & $12(52.2)$ & & \\
\hline M694V heterozygote & $6(26.1)$ & & \\
\hline \multicolumn{4}{|l|}{ E148Q heterozygote } \\
\hline M680I homozygote & $2(8.7)$ & & \\
\hline None & $3(13.0)$ & & \\
\hline ESR & $8.69(1 \sim 23)$ & & \\
\hline CRP & $1.17(0.09 \sim 5.45)$ & & \\
\hline Fibrinogen & $250.38(120 \sim 385)$ & & \\
\hline
\end{tabular}

Values are presented as mean \pm standard deviation, number $(\%)$ or mean (min $\sim$ max). FMF: Familial Mediterranean fever, ESR: erythrocyte sedimentation rate, CRP: C-reactive protein. $\mathrm{p}<0.05$ is statistically significant. 
least 23 patients for each group would be needed to obtain an approximately 90 percent power with $\mathrm{d}=0.86$ effect size, $\alpha=0.05$ type I error and $\beta=0.10$ type II error. Effect size was calculated according to mean values and standart deviations of the results of TBUT test in Karalezli et al.'s study [16].

\section{RESULTS}

The demographic features of the patients and healthy volunteers are presented in the Table 1 . All of the patients with FMF were under colchicine medication, and one of the FMF patients was using additional anakinra therapy. Episcleritis, keratitis, uveitis (anterior, intermediate, posterior or panuveitis) or retinal pathology was not noted in any of the participants. There was no statistically significant difference between the demographic features of two groups (all $\mathrm{p}>0.05$ ).

There was no statistically significant difference of Schirmer-I, TBUT, and OSDI scores between the patients with FMF and control group (all p>0.05) (Table 2).

In the patients with FMF, no statistically significant correlation were found between acute phase reactants (ESR, CRP and fibrinogen levels) and xerophthalmia tests scores (Schirmer-I, TBUT, OSDI) (all p >0.05) (Table 3). Additionally, no correlation was shown between the xerophthalmia tests scores (Schirmer-I, TBUT, OSDI) and disease duration or the number of attacks (all $\mathrm{p}>0.05$ ) (Table 3). Furthermore, the positivity of FMF genes was not correlated with xerophthalmia tests scores (Schirmer-I, TBUT, OSDI) either (all $\mathrm{p}>0.05$ ).

\section{DISCUSSION}

The primary aim of this study was to investigate whether FMF would cause DES because it is an autoinflammatory disease. According to this hypothesis, we tried to evaluate if there is a relationship between these two entities. But no association was found between FMF and DES in the present study.

In the literature there are only limited studies about DES in patients with FMF. In a study conducted by Karalezli et al. [16] the disruptions in tear-film functions and the ocular surface characteristics of FMF patients were compared with healthy controls. For testing the tear functions, they used Schirmer-I and BUT tests. Corneal fluorescein, Rose Bengal staining and conjunctival impression cytology were also carried out. They also used a 4-point Likert scale

Table 2. Comparison of xerophthalmia tests of the patients and healthy controls

\begin{tabular}{lccc}
\hline \multicolumn{1}{c}{ Variable } & FMF patients $(\mathrm{n}=23)$ & Healthy controls $(\mathrm{n}=23)$ & $\mathrm{p}$-value \\
\hline Schirmer-I test $(\mathrm{mm} / 5 \mathrm{~min})$ & $14.78 \pm 8.38$ & $13.09 \pm 10.54$ & 0.276 \\
OSDI & $9.04 \pm 2.75$ & $10.86 \pm 1.77$ & 0.773 \\
TBUT (s) & $14.69 \pm 5.32$ & $15 \pm 6.45$ & 0.698 \\
\hline
\end{tabular}

Values are presented as mean \pm standard deviation. FMF: Familial Mediterranean fever, OSDI: ocular surface disease index, TBUT: tear break-up time. $\mathrm{p}<0.05$ is statistically significant.

Table 3. The correlation between some laboratory and clinical parameters and xerophthalmia tests scores

\begin{tabular}{lccrr}
\hline \hline \multirow{2}{*}{ Variable } & & Schirmer-I & TBUT & OSDI \\
\hline \multirow{2}{*}{ CRP } & $\mathrm{R}$ & 0.807 & 0.355 & 0.754 \\
\multirow{2}{*}{ Fibrinogen } & $\mathrm{P}$ & 0.06 & 0.102 & 0.098 \\
\multirow{2}{*}{ Disease duration } & $\mathrm{R}$ & -0.180 & -0.279 & -0.147 \\
\multirow{2}{*}{ Number of attacks } & $\mathrm{P}$ & 0.190 & 0.780 & 0.080 \\
& $\mathrm{R}$ & -0.332 & -0.101 & -0.560 \\
& $\mathrm{P}$ & 0.122 & 0.098 & 0.070 \\
& $\mathrm{R}$ & 0.166 & 0.178 & 0.064 \\
& $\mathrm{P}$ & 0.449 & 0.210 & 0.100 \\
& $\mathrm{R}$ & -0.315 & -0.122 & 0.760 \\
\hline
\end{tabular}

TBUT: tear break-uptime, OSDI: ocular surface disease index, ESR: erythrocyte sedimentation rate, CRP: C-reactive protein level, R: correlation, P: p-value. $p<0.05$ is statistically significant. 
for assessing subjective symptoms such as foreign-body sensation, burning, stinging and ocular fatigue. Karalezli et al. [16] found the mean Schirmer-I values of FMF patients did not differ from those of normal controls. However, abnormal BUT test results, abnormal fluorescein and Rose Bengal staining and the existence of subjective ocular complaints were more frequent in FMF patients than in controls. They claimed that, those findings suggest that tear production is not changed in FMF patients, but tear quality is markedly affected. But in the present study, both Schirmer-I and TBUT test results and OSDI scores which also evaluates subjective complaints were not statistically different from control groups. In the previous study [16], impression cytology analysis provided evidence that compared to control patients, people with FMF had significant squamous metaplasia and goblet cell loss. These findings support the existence of an ocular surface disease in patients with FMF, which is probably the result of the inflammatory nature of FMF disease. Low-grade inflammation in the FMF can be a result of goblet cell destruction and reduced tear film quality, although no mention was made of whether the patient or the control group had been undergone treatment that could affect DES or the use of colchicine. Also in a scientific letter written by Lazar et al. [21] about the previous study [16], because colchicine can affect the ocular surface; the authors presume that the changes observed in the ocular surface may have been secondary to the colchicine treatment. In the literature; delay of corneal wound healing in patients treated with colchicine had been reported $[22,23]$. In a study aimed to determine whether detectable concentrations of colchicine were present in the tear fluid of treated patients with FMF, it was shown that colchicine, given systemically, reaches the corneal surface and thus gives credence to the possible inhibitory effect of this drug on corneal wound healing in the cases described in the literature [24]. In the present study the mean time of the disease diagnosis was $8.19 \pm 9.34$ years, so the patients in this study were under the colchicine treatment approximately for about 8 years. In the study of Karalezli et al. [16], there were no information about disease duration or the drug history of patients. Therefore, the differences between the results of present study and Karalezli's study may be due to this.

In the present study, apart from the investigation about DES; all of the participants underwent a detailed ophthalmologic examination also. None of the FMF patients had episcleritis, uveitis (anterior, intermediate, posterior or panuveitis), or a retinal pathology. In FMF, ophthalmic involvement is rare and nonspecific, in contrast to other autoinflammatory disorders, and has been documented as case reports [4-7]. Most cases responded to colchicine treatment and recovery was experienced after starting topical and systemic antiinflammatory medications. Salehzadeh et al. [6] examined 12 patients who were diagnosed idiopathic uveitis. None of the patients had positive findings in terms of clinical findings of FMF and MEVF gene mutation. They concluded that FMF could not be considered as an underlying cause of idiopathic uveitis. In a study conducted by Yazici et al. [7], the archive of a tertiary referral center's uvea division consisting of 1,265 uveitis patients was reviewed and the data of only 6 patients with FMF diagnosis were gathered. They concluded that, ocular involvement of FMF should be considered as a diagnosis of exclusion after all possible underlying causes are eliminated.

Coexistence of FMF with other inflammatory diseases such as RA, Sjögren's syndrome (SS), anklosying spondilitis, systemic lupus erythematosus and juvenile idiopathic arthritis has been reported [25-29].The association of SS and RA with DES is well-known [8] and FMF related ocular findings such as uveitis, iritis, iridocyclitis may also be related to the coexistence of FMF with those diseases. However, there is some evidence about the concurrence of FMF and, SS which one of the findings is DES. Increased IL-18 levels in the patients with FMF was presented in two case report $[26,30]$. Higher IL-18 level detected in patients with concomitant SS and FMF, and reported that FMF-related dysregulated IL-18 production and chronic inflammation might be linked with the occurrence of SS. But; none of the patients with FMF in the present study had a concomitant inflammatory disease.

IL-1 receptor blockade have been shown in the treatment of DES [13]. Amparo et al. [31] published favorable results with Anakinra (recombinant, nonglycosylated human interleukin-1 receptor antagonist [IL-1Ra]) as an eye drop in medical treatment of DES [31]. Moreover topical mesenchymal stem cells (MSC) has been performed in dogs with DES also it has been reported that MSC leads decrease in inflammatory markers (IL-6, IL-1, and TNF- $\alpha$ ) and MSC could be an alternative treatment regime in DES [32]. MSC application and topical IL-1 blockers in DES treatment may be used commonly in near future.

In addition, DES can be related to the other conditions including use of computer and smart devices, which is very common among population [33]. Therefore this fact 
might have also contributed to the result that the frequency of DES was not found to be significantly different between patient and control group. We did not note the duration of computer and smart devices use in patient and control groups and this can be a limitation of the study.

There are also some other limitations of the present study. Although the number of patients to be included in the study was determined by pre-study power analysis; future studies with more patients may also have different results. Secondly, no examination of the patients was made during an FMF attack. Additionally, there were no newly diagnosed patients, all of the patients were under colchicine treatment. Since this study was performed in a rural area of Turkey, ethnic variations were not noted. This is another limitation of the present study.

\section{CONCLUSION}

In conclusion, FMF was not found to be associated with DES and no any other eye manifestations have been found to be related with FMF.

\section{CONFLICT OF INTEREST}

No potential conflict of interest relevant to this article was reported.

\section{AUTHOR CONTRIBUTIONS}

S.S. was involved in conception and design of study, acquisition of data, drafting the manuscript, revising the manuscript critically for important intellectual content. A.K. was involved in acquisition of data, drafting the manuscript. E.E.K. analysis and/or interpretation of data. F.A.K. was involved in conception and design of study, acquisition of data. H.R.E. was involved in acquisition of data.

\section{REFERENCES}

1. Samuels J, Ozen S. Familial Mediterranean fever and the other autoinflammatory syndromes: evaluation of the patient with recurrent fever. Curr Opin Rheumatol 2006;18: 108-17.

2. Sarı İ, Birlik M, Kasifoğlu T. Familial Mediterranean fever: an updated review. Eur J Rheumatol 2014;1:21-33.

3. Tunca M, Akar S, Onen F, Ozdogan H, Kasapcopur O, Yalcinkaya F, et al.; Turkish FMF Study Group. Familial Mediterranean fever (FMF) in Turkey: results of a nationwide multicenter study. Medicine (Baltimore) 2005;84:
1-11.

4. Petrushkin H, Stanford M, Fortune F, Jawad AS. Clinical review: familial Mediterranean fever-an overview of pathogenesis, symptoms, ocular manifestations, and treatment. Ocul Immunol Inflamm 2016;24:422-30.

5. Tarabishy AB, Hise AG, Traboulsi EI. Ocular manifestations of the autoinflammatory syndromes. Ophthalmic Genet 2012;33:179-86.

6. Salehzadeh F, Yasrebi O, Hosseini Khotbesara M, Hosseini Khotbesara M. Idiopathic uveitis and familial Mediterranean fever: is there any relationship? Autoimmune Dis 2014; 2014:238931.

7. Yazici A, Ozdal P, Yuksekkaya P, Elgin U, Teke MY, Sari E. Ophthalmic manifestations in familial Mediterranean fever: a case series of 6 patients. Eur J Ophthalmol 2014;24:593-8.

8. Zoukhri D. Effect of inflammation on lacrimal gland function. Exp Eye Res 2006;82:885-98.

9. Yang XO, Panopoulos AD, Nurieva R, Chang SH, Wang D, Watowich SS, et al. STAT3 regulates cytokine-mediated generation of inflammatory helper T cells. J Biol Chem 2007;282:9358-63.

10. Mankan AK, Kubarenko A, Hornung V. Immunology in clinic review series; focus on autoinflammatory diseases: inflammasomes: mechanisms of activation. Clin Exp Immunol 2012;167:369-81.

11. Direskeneli H, Ozdogan H, Korkmaz C, Akoglu T, Yazici H. Serum soluble intercellular adhesion molecule 1 and interleukin 8 levels in familial Mediterranean fever. J Rheumatol 1999;26:1983-6.

12. Huang JF, Zhang Y, Rittenhouse KD, Pickering EH, McDowell MT. Evaluations of tear protein markers in dry eye disease: repeatability of measurement and correlation with disease. Invest Ophthalmol Vis Sci 2012;53:4556-64.

13. Okanobo A, Chauhan SK, Dastjerdi MH, Kodati S, Dana R. Efficacy of topical blockade of interleukin-1 in experimental dry eye disease. Am J Ophthalmol 2012;154:63-71.

14. Ben-Zvi I, Livneh A. Colchicine failure in familial Mediterranean fever and potential alternatives: embarking on the Anakinra trial. Isr Med Assoc J 2014;16:271-3.

15. Türkyilmaz K, Küçükali Türkyilmaz A, Kurt A, Kurt EE, Sevim MŞ, Oner V. Investigation of tear osmolarity in early rheumatoid arthritis: relation to disease activity. Can J Ophthalmol 2013;48:235-9.

16. Karalezli A, Borazan M, Yilmaz S, Kiyici H, Akova YA. Conjunctival impression cytology and tear-film changes in patients with familial Mediterranean fever. Acta Ophthalmol 2009;87:39-43.

17. Livneh A, Langevitz P, Zemer D, Zaks N, Kees S, Lidar T, et al. Criteria for the diagnosis of familial Mediterranean fever. Arthritis Rheum 1997;40:1879-85.

18. The definition and classification of dry eye disease: report of the Definition and Classification Subcommittee of the International Dry Eye WorkShop (2007). Ocul Surf 2007;5: 75-92.

19. Schiffman RM, Christianson MD, Jacobsen G, Hirsch JD, Reis BL. Reliability and validity of the Ocular Surface Disease Index. Arch Ophthalmol 2000;118:615-21.

20. Irkec MT; Turkish OSDI Study Group. Reliability and validity of Turkish translation of the Ocular Surface Disease Index (OSDI) in dry eye syndrome. Investig Ophthalmol Vis Sci 2007;48:408. 
21. Lazar M, Rothkoff L. Colchicine and ocular surface changes in familial Mediterranean fever. Acta Ophthalmol 2010; 88:e5; author reply e6.

22. Alster Y, Varssano D, Loewenstein A, Lazar M. Delay of corneal wound healing in patients treated with colchicine. Ophthalmology 1997;104:118-9.

23. Leibovitch I, Alster Y, Lazar M, Langevitz P, Livneh A, Loewenstein A. Corneal wound healing in a patient treated with colchicine for familial Mediterranean fever (FMF). Rheumatology (Oxford) 2003;42:1021-2.

24. Leibovitch I, Alster Y, Scherrmann JM, Azmon B, Barequet IS, Livneh A, et al. Colchicine in tear fluid of treated patients with familial Mediterranean fever. Cornea 2003;22:191-3.

25. Mori S, Yonemura K, Migita K. Familial Mediterranean fever occurring in an elderly Japanese woman with recent-onset rheumatoid arthritis. Intern Med 2013;52:385-8.

26. Tanaka M, Migita K, Miyashita T, Maeda Y, Nakamura M, Komori A, et al. Coexistence of familial Mediterranean fever and Sjögren's syndrome in a Japanese patient. Clin Exp Rheumatol 2007;25:792.

27. Akkoc N, Sari I, Akar S, Binicier O, Thomas MG, Weale ME, et al. Increased prevalence of M694V in patients with ankylosing spondylitis: additional evidence for a link with familial Mediterranean fever. Arthritis Rheum 2010;62:3059-63.
28. Yildiz G, Kayatas M, Uygun Y, Timuçin M, Candan F. Coexistence of systemic lupus erythematosus and familial Mediterranean fever. Intern Med 2010;49:767-9.

29. Kaya S, Kaptanoglu E, Elden H, Hizmetli S. Coexistence of familial Mediterranean fever and juvenile idiopathic arthritis with osteoporosis successfully treated with etanercept. Intern Med 2010;49:619-22.

30. Haznedaroglu S, Oztürk MA, Sancak B, Goker B, Onat AM, Bukan N, et al. Serum interleukin 17 and interleukin 18 levels in familial Mediterranean fever. Clin Exp Rheumatol 2005;23 (4 Suppl 38):S77-80.

31. Amparo F, Dastjerdi MH, Okanobo A, Ferrari G, Smaga L, Hamrah $\mathrm{P}$, et al. Topical interleukin 1 receptor antagonist for treatment of dry eye disease: a randomized clinical trial. JAMA Ophthalmol 2013;131:715-23.

32. Sgrignoli MR, Silva DA, Nascimento FF, Sgrignoli DAM, Nai GA, da Silva MG, et al. Reduction in the inflammatory markers CD4, IL-1, IL- 6 and TNF $\alpha$ in dogs with keratoconjunctivitis sicca treated topically with mesenchymal stem cells. Stem Cell Res 2019;39:101525

33. Kim DJ, Lim CY, Gu N, Park CY. Visual fatigue induced by viewing a tablet computer with a high-resolution display. Korean J Ophthalmol 2017;31:388-93. 